\title{
Educational software simulation for fiber optic dispersion
}

Gustavo Medina Arredondo, Mario Yepes Cifuentes, Samuel Jaramillo Florez

Gustavo Adolfo Medina Arredondo, Mario Andres Yepes Cifuentes, Samuel Angel Jaramillo Florez, "Educational software simulation for fiber optic dispersion," Proc. SPIE 2525, 1995 International Conference on Education in Optics, (13 October 1995); doi: 10.1117/12.224048

SDIE Event: SPIE's 1995 International Symposium on Optical Science, Engineering, and Instrumentation, 1995, San Diego, CA, United States 


\title{
Educational Software Simulation for Fiber Optic Dispersion
}

Gustavo A. Medina, Mario A. Yepes, Samuel A. Jaramillo.

Universidad Pontificia Bolivariana, Optronics Group.

\begin{abstract}
$\underline{\text { ABSTRACT }}$
The fiber optic as all the other transmission lines, presents some problems like the deformation and the attenuation of signals. Even thougt these inconvinients are of little magnitude, it's important to keep them in mind, specialy if we are studding the optic fiber in high frequency transmissions and in long distances. In the following we present a sof tware designed to calculate and make a graphic comparison of the dispersion in the optic fiber, either if its monomode or multimode.
\end{abstract}

Keywords: Optic fibrrs, ligth dispersion, Optic disprsion,

\section{DISPERSION IN OPTIC FIBERS}

The dispersion in a transmission line its a concept used to describe the differences in time propagation of the waves in the transmission line. In the case of the optic fiber, the differences in time propagation come from the fact that the light phase propagation ( and the propagation time of the wave), depends on the wave length.

The light pulses travels thruogh the Optic fiber with a group velocity. It's expression is:

$c_{g}=\frac{c}{n_{8}}$

Where $n_{g}$ it's the group refraction index inside de nucleus, $n_{g}$ it's also a function of the wave length

( $\lambda$ ). The time a wave takes to cover certain distance is:

$t_{g}=\frac{L}{c_{g}}=\frac{L}{c} n_{g}$

Because de time it's a function of the refraction index, and the refraction index it's a function of $\lambda$, the time depends of $\lambda$.

Most light surces have a spectral with $(\Delta \lambda)$ distributed around a central $\lambda$. Because of this, The different component of a light source travels at diverse speeds Trough the optic fiber at different times.

Three factors are the responsibles of dispersion in an optic fiber:

- Modal dispersion $\left(\sigma_{m}\right)$.

- Material dispersion $\left(m_{o}\right)$.

- wavegide dispersion $\left(\sigma_{g}\right)$.

The first two are important in the Multimode fibers, the last two have importance in the monomode fiber. There is another kind of dispersion, The chromatic dispersion. This dispersion it's equal to the trigonometric addition of the material dispersion and the wavegide dispersion.

\section{MODAL DISPERSION.}

The modal dispersion in an optic fiber occurs because the different modes that travel trought the waveguide take different directions inside the nucleus, on the other hand, the light speed in a certain point inside the fiber depends on the refraction index. This tow effects together causes retards in the different propagation modes making the light pulse more wide. 
the matematic expression for the modal dispersion is:

$$
\sigma_{m}=\frac{n_{1}^{2}-n_{2}^{2}}{2 n_{1} c}
$$

Were:

$c=$ light speed,

$n_{1}=$ refraction index of the nucleus.

$n_{2}=$ refraction index of the revestiment.

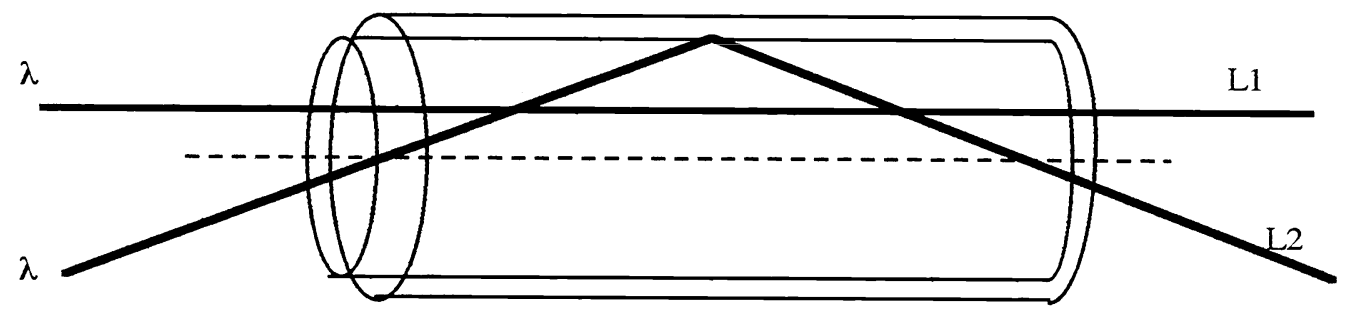

Here we see how two light beams travels trough diferent ways.

To add the modal dispersion of several sections, we use:

$\sigma_{m, T} \frac{1}{\gamma}=\sum_{i=1}^{N} \sigma_{m, i}^{\frac{1}{\gamma}}$

$\mathrm{N}=$ number of fibers.

$\sigma_{m, i}=$ modal dispersion of each fiber.

$\gamma=$ concatenation factor.

The common value of $y$ is a little less than 1 .

\section{MATERIAL DISPERSION}

The material dispersion depends directly from the light suorce. To be more specific, the material dispersion is caused by the spectral with of the source $(\Delta \lambda)$.

We just saw in the firsts paragraphs who the refraction index depends of $\lambda$ an who this dependence causes diferences in the times of propagation. If the light source have a little $\Delta \lambda$ the material dispersion will be shorter, but of the light source has a big $\Delta \lambda$, the material dispersion will be larger.

The material dispersion it's a measurement of the variation of the group refraction index .

$m_{o}(\lambda)=\frac{d n_{g}(\lambda)}{c \cdot d \lambda}=\frac{d t_{g}(\lambda)}{L \cdot d \lambda}$

The material dispersion can be modified changing the amount of doping substance in the fiber.

Another formula for the material dispersion (the most used) is:

$m_{o}=L \frac{\lambda}{c} \Delta \lambda\left(\frac{d^{2} n_{1}}{d \lambda^{2}}\right)=\Delta \lambda M(\lambda) L$ 
Were.

$\mathrm{L}=$ longitude of the waveguide.

$c=$ light speed.

$\Delta \lambda=$ quadratic half of the light spectral with of the light source.

$\lambda=$ wave length

$\mathrm{M}=$ material dispersion index.

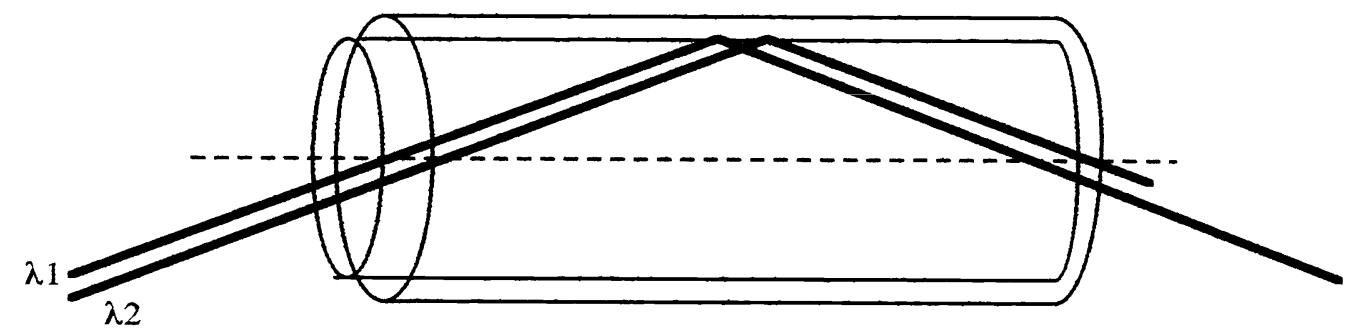

Here we have two light beams with two $\lambda$ and two speeds.

For most optic fibbers the $M(\lambda)$ it's insignificant when $\lambda=1300 \mathrm{~nm}$.

\section{WAVEGUIDEDISPERSION}

The waveguide dispersion have a similitude to the material dispersion only that the waveguide dispersion is modified by the optic or geometric attributes of the fiber not by the doping elements.

The waveguide dispersion can be expressed by the next formula:

$\sigma_{g} \approx-\left(\frac{\Delta \lambda L}{4 \pi^{2} n_{1} c}\right)\left(\frac{\lambda}{a^{2}}\right)$

Where:

$\mathrm{a}=$ nucleus radium

$n_{1}=$ refraction index of the nucleus.

The addition of the tow dispersions just considered( wavegide and material dispersion ) is named cromatic dispersion.

$\sigma_{c}(\lambda)=m_{o}+\sigma_{g}(\lambda)$

The total amount of dispersion in an optic fiber is:

$\sigma_{t}=\sqrt{\sigma_{m}^{2}+\left(m_{o}+\sigma_{g}\right)^{2}}$

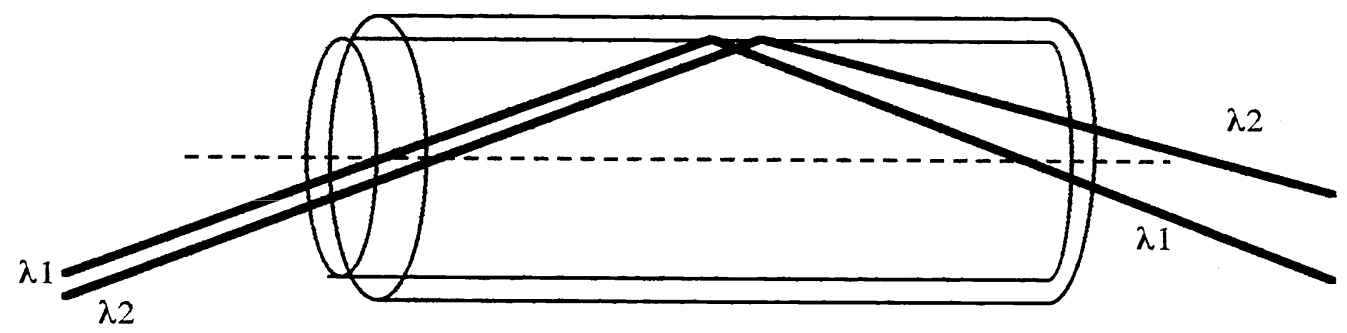

Because of the geometric form of the waveguide, the two light beams take different ways. 


\section{MATEMATIC}

To make this formulas really functional its necessary to find a matematic relation between $\lambda$ and the refraction index. Unfortunately there is no such a thing, there are only some graphic relations with no tabulation. That's why we were forced to make a precise tabulation of this graphics.

But there is another problem yet, the optic fiber is made from silica Crystal, doped with different amounts of different dopants so the refraction index changes not only depends on the working wave length but also if the amount, and the kind, of dopant in the fiber is different, so we had to find a relation between this two parameters and $\lambda$. This relation doesn't really exist, and there is no way it can be expressed by a matematic formula. This fact represent a big problem in the dispersion equations (and even a bigger problem if you want to derive in cases like the material dispersion) that why we had to use numeric approximations .

\section{THE SOFTWARE.}

The program used to calculate the dispersion in an optic fiber is called FIBER and it's made in the Borland Pascal package 7.0. The program it's friendly (for easy use) and has an accessible help menu.

The system requirements for the program are: chip 386 or later and D.O.S.. All the extensions of the program has to be in the same directory .

the extensions of the program are:

Fiber .exe: executable folder.

Fiberhlp.hlp: help folder.

Egavga.bgi: graphic interface unit.

Fiber.doc: source code.

Trip.chr: font source.

To run the program it's just necessary to run fiber.exe .

All the information necessary to run the program it's in the help menu and the graphic interfaces of the program.

A graphic example of the disperssion of a light pulse in an optic fiber will be the next graphic. In the graphic we can see a simplified aproximation of what the program does.

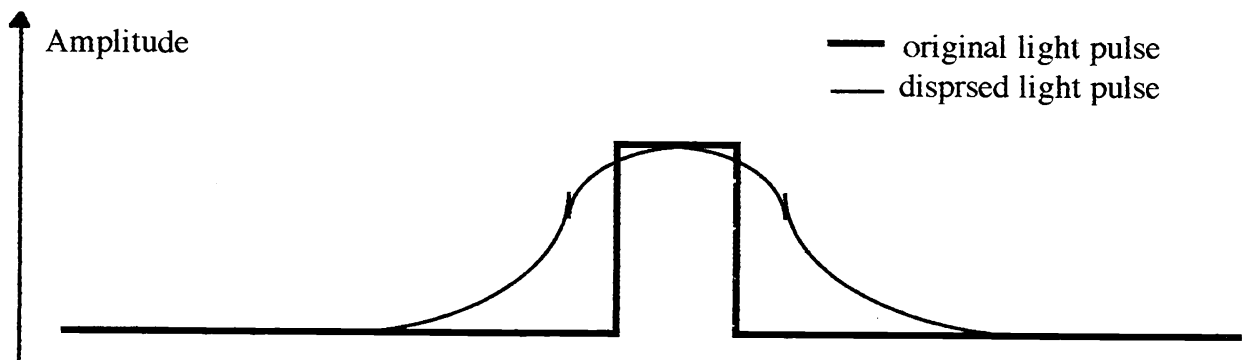

\section{REFERENCES}

J.F., Samuel Ángel. "Comunicación por Fibras Ópticas;Nivel I introductorio."

FACOMEC. "Fibras Ópticas."

KAO, C. K. "Optical Fiber".

J. F., Samuel Ángel. "Redes de Telecomunicaciones;Comunicaciones por fibra optica." 